\title{
Polarization splitting in organic-based microcavities working in the strong coupling regime
}

\author{
Andrea Camposeo ${ }^{\mathrm{a}, *}$, Luana Persano ${ }^{\mathrm{a}}$, Pompilio Del Carro ${ }^{\mathrm{a}}$, \\ Tersilla Virgili ${ }^{b}$, Roberto Cingolani ${ }^{\text {a }}$, Dario Pisignano ${ }^{\mathrm{a}}$ \\ ${ }^{a}$ National Nanotechnology Laboratory of CNR-INFM, Distretto Tecnologico ISUFI, Università di Lecce, via Arnesano, I-73100 Lecce, Italy \\ b IFN-CNR, clo Dipartimento di Fisica, Politecnico di Milano, P.zza Leonardo da Vinci 32, 20132 Milano, Italy
}

Received 1 February 2006; received in revised form 21 May 2006; accepted 22 June 2006

Available online 20 July 2006

\begin{abstract}
The emission features and the polarization splitting are investigated in organic microcavities operating in the strong coupling regime. The microcavity relies on a $J$-aggregate of cyanine dyes as active layer, and on high reflectivity distributed Bragg reflectors, fabricated by low-temperature reactive electron-beam evaporation. The operation of the microcavities in the strong coupling regime is demonstrated both by reflectivity and photoluminescence characterization. A vacuum Rabi splitting in the range $30-100 \mathrm{meV}$ is reported. Important, both polariton branches exhibit a TM-TE polarization splitting depending on the incident angle, $\theta$, and exhibiting a maximum of $35 \mathrm{meV}$. The angular behaviour of the splitting in the strong coupling regime is investigated and described by the $\sin ^{2} \theta$ dependence predicted by the polarization splitting model. (c) 2006 Elsevier B.V. All rights reserved.
\end{abstract}

PACS: $42.50 . \mathrm{Fx} ;$ 78.40.Me; 78.66.Qn; 78.67.Pt

Keywords: Organic Microcavity; Strong coupling; Polarization splitting

\section{Introduction}

Organic semiconductor microcavities, a class of devices in which a semiconductor conjugated layer is embedded between two mirrors, have attracted a lot of attention both from the fundamental and the practical interest viewpoint, for their possible technological applications in advanced optoelectronics [1-3]. In such devices, the optical properties of the

\footnotetext{
* Corresponding author. Tel.: +390832298147; fax: +390832 298238.

E-mail address: andrea.camposeo@unile.it (A. Camposeo).
}

active semiconductor layer can be dramatically altered due to quantum electrodynamics (QED) effects [4]. In fact, the light-matter interaction resulting in the absorption and emission of photons does not only depend on the electronic properties of the active molecules, but also on the intensity of the electric field of the incident light, which is determined by the photonic boundary conditions. Among the numerous methods allowing a modification of the boundary conditions (i.e. the control of the resulting emission), the exploitation of photonic crystals is particularly interesting, because it can provide strong confinement of photons in a number of 
dimensions [5]. In particular, one-dimensional photonic crystals, such as vertical microcavities, are the simplest systems where QED effects are observable. The interactions between an organic or inorganic semiconductor in a microcavity and an electromagnetic field can occur under two different regimes, namely, the weak and the strong coupling, respectively. In the strong coupling regime, a cavity-photon couples strongly to an exciton having the same energy and in-plane momentum. The coherent coupled state thus formed is termed a cavity polariton, and can be considered as a mixture of the exciton and cavity-photon modes.

Recently, the strong coupling regime between exciton and photon modes has also been demonstrated for microcavities containing organic materials with large exciton oscillator strengths and relatively narrow line widths, such as porphyrin [6], $J$-aggregated cyanine dyes [7], conjugated polymers [8] and polycrystalline films of small organic molecules [9]. The occurrence of strong coupling is easily identified by the observation of an anticrossing between the cavity photon and the exciton modes that ideally results in resonance in a doublet of cavity polariton states. These exhibit equal linewidth and intensity and are separated by the vacuum Rabi-splitting energy [10,11].

So far, extensive studies have been performed for both inorganic [10] and organic cavity polaritons [12]. In particular, the development of novel architectures exhibiting strong coupling with organics has great importance for the realization of organic optoelectronic devices with featured characteristics [13]. Recently, the enhancement of the optical nonlinearity was observed in a transparent microcavity with all-dielectric mirrors and a $J$-aggregate of cyanine dyes embedded [14] and high-finesse organic microcavities with DBR mirrors have been realized which show quality factors in the range 440-620 [15]. In this work, we report on the fabrication and characterization of an organic semiconductor symmetric microcavity with dielectric mirrors, operating in the strong coupling regime. A novel lowtemperature fabrication technique of distributed Bragg reflectors (DBR), that we successfully exploited for the fabrication of vertical cavity organic lasers [16], was applied to the realization of a microcavity with a $J$-aggregate of cyanine dyes as active layer. This organic material is known to have the necessary optical properties for strong coupling operation $[7,12]$. The devices we fabricated are characterized by a vacuum Rabi splitting in the range $30-100 \mathrm{meV}$ and exhibit a polarization splitting up to $35 \mathrm{meV}$. Although polarization effects has been extensively studied in GaAs-based microcavites [17] and birefringence-induced polarization splitting was previously observed in microcavities with $J$-aggregates active layer [18], the present work is, to the best of our knowledge, the first extensive experimental investigation of this effect and of its angular behaviour in organic-based microcavities. In particular, we found that both the mode splitting within the lower (LP) and the upper (UP) polariton branch are well described by the $\sin ^{2}(\theta)$ angular dependence predicted by the polarization splitting model, for small $(0 \leqslant \theta \leqslant 20)$ and for large $(\theta \leqslant 35)$ angles, respectively.

\section{Experimental}

A scheme of the microcavities is shown in the inset of Fig. 1(b). The technique we developed for the DBRs fabrication has been described in details elsewhere [19] and will be here briefly recalled. The bottom DBR, deposited onto Corning glass substrates is composed by 5.5 pairs of $\mathrm{SiO}_{2}$ $\left(d_{1}=127 \mathrm{~nm}\right)$ and $\mathrm{TiO}_{2}\left(d_{2}=83 \mathrm{~nm}\right)$ layers, used as low- $\left(n_{1}=1.41\right)$ and high- $\left(n_{2}=1.98\right)$ refractive index medium, respectively. The oxide films were deposited from $99.9 \%$ purity $\mathrm{TiO}_{2}$ tablets and $\mathrm{SiO}_{2}$ disks (Leybold, Germany) at a working pressure of $2.4 \times 10^{-4}$ mbar. A controlled oxygen atmosphere was accomplished by a gas inlet through a precision needle valve. A blend of an inert transparent matrix polymer (polyvinyl alcohol, PVA) containing a $10 \mathrm{wt} . \%$ cyanine dye (Hayashibara Biochemical Labs Inc.) was spin coated on top of the DBR mirror. This blend was spun from a 1:1 mixture of methanol and deionised water. Fig. 1(a) shows the absorption and emission spectrum of the $J$-aggregate forming cyanine dye $2,2^{\prime}$ dimethyl-8-phenyl-5,6,5',6'-dibenzothiacarbocyanine chloride. The $J$-aggregate shows a strong absorption peak at $1.78 \mathrm{eV}$ with a linewidth of $70 \mathrm{meV}$. The top mirror, composed by 8.5 pairs was then evaporated on the cavity layer. $\mathrm{SiO}_{x}\left(d_{1}=127 \mathrm{~nm}\right)$ and $\mathrm{TiO}_{x}$ $\left(d_{1}=87 \mathrm{~nm}\right)$ films were deposited at low temperature $\left(<70{ }^{\circ} \mathrm{C}\right)$, thus preventing any deleterious effect on the optical properties of the active layer. We estimated a quality factor of the empty cavity of 400 by a transfer matrix reflectivity (TMR) model based on the measured mirrors reflectivities. The absorption-reflection properties of the devices at normal incidence were measured by a Cary 5000 

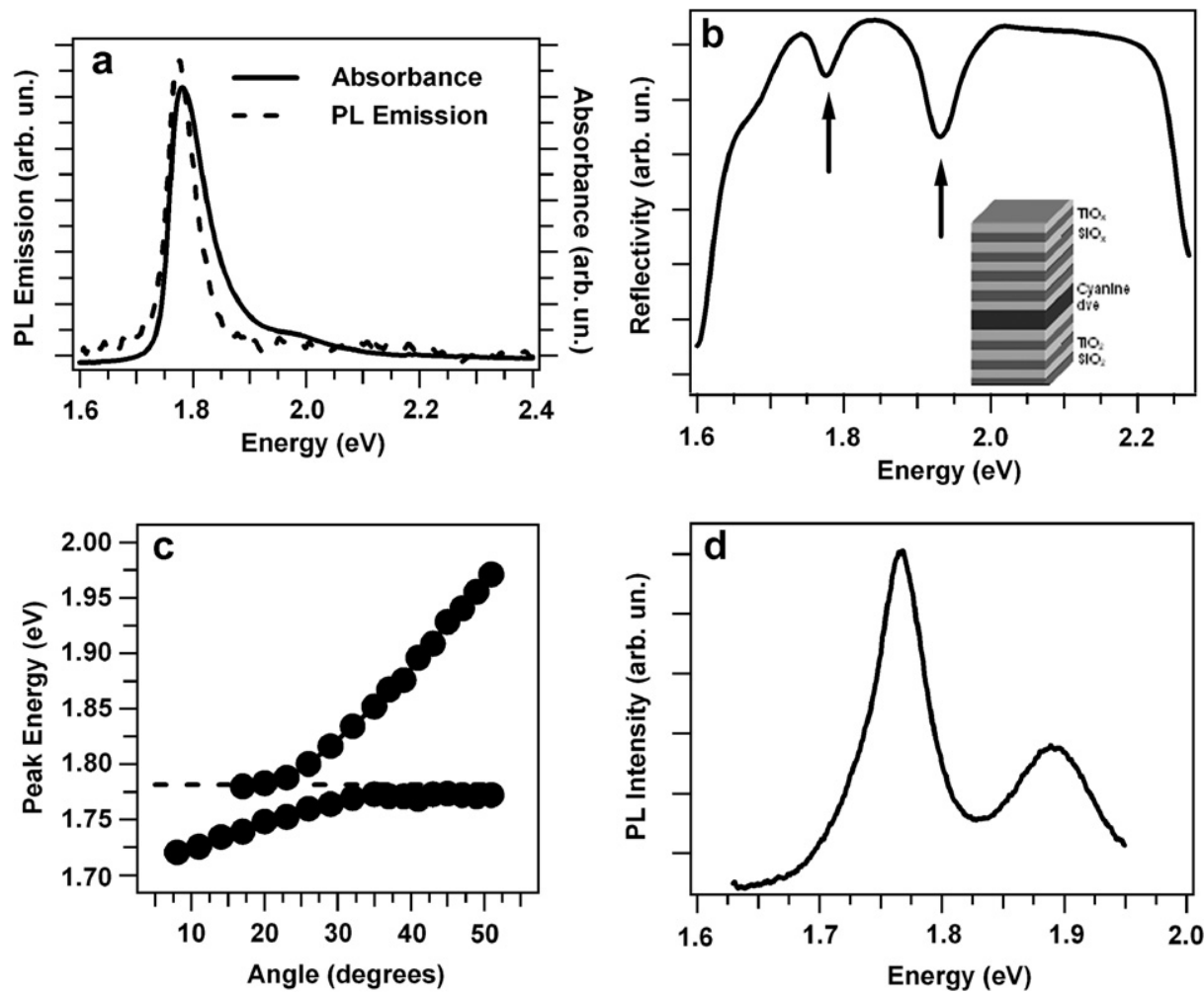

Fig. 1. (a) Absorbance and PL emission of a bare film of $J$-aggregated forming cyanine dye, used in this work. (b) Cavity reflectivity recordered at $45^{\circ}$ as a function of incident light energy. The arrows mark the energy of the LP and UP polariton. In the inset a scheme of the monolithic microcavity is reported. (c) Dispersion curves as a function of the incident angle, obtained by reflectivity measurements. The dashed horizontal line indicates the energy of $1.78 \mathrm{eV}$ of a bare film of $J$-aggregate cyanine dye. (d) Photoluminescence spectrum of the cavity excited by a cw He-Ne laser. The sample was excited at normal incidence and photoluminescence recordered at $42^{\circ}$.

spectrophotometer (Varian, Australia). Angleresolved reflection spectra were obtained by illuminating the samples, mounted on a rotating holder, with a white $\mathrm{W}$ halogen lamp and recording the spectrum of the reflected light by a monochromator coupled to a CCD detector. The photoluminescence of the samples was measured by exciting the cavities with a $\mathrm{He}-\mathrm{Ne} \mathrm{cw}$ laser (excitation energy $1.959 \mathrm{eV}$ ), with a typical emitting power of $0.3 \mathrm{~mW}$ in order to avoid saturation effects [20]. High contrast polarization filters were used to analyze the polarization state of the emitted light. All measurements were carried out at room temperature, in air atmosphere.

\section{Results and discussion}

A typical reflection spectrum of the fabricated monolithic cavities is displayed in Fig. 1(b), showing the presence of cavity polaritons. The two peaks evidenced by arrows in Fig. 1(b) correspond to the
LP and UP polariton branch, respectively, as also indicated by photoluminesence measurements (Fig. 1(d)). The effective strong coupling between the exciton and the cavity-photon modes is indeed confirmed by the characteristic anticrossing behaviour of the two coupled modes dispersion (Fig. 1(c)), obtained by reflectivity angle-tuning measurements. A similar trend was also observed for the photoluminescence measurements. In fact, the cavity mode exhibits an angle dependent resonant energy:

$E_{\mathrm{c}}(\theta)=E_{0}\left(1-\frac{\sin ^{2}(\theta)}{n_{\mathrm{eff}}}\right)^{-1 / 2}$

In the previous expression, $E_{0}=h c / n_{\mathrm{eff}} L_{\mathrm{c}}$ stands for the photon energy at normal incidence if there is no phase delay in the mirrors, $L_{\mathrm{c}}$ is the cavity thickness and $n_{\text {eff }}$ is an effective refractive index of the microcavity system.

Fig. 2 shows the reflectivity spectra (at normal incidence) of three monolithic cavities upon chang- 


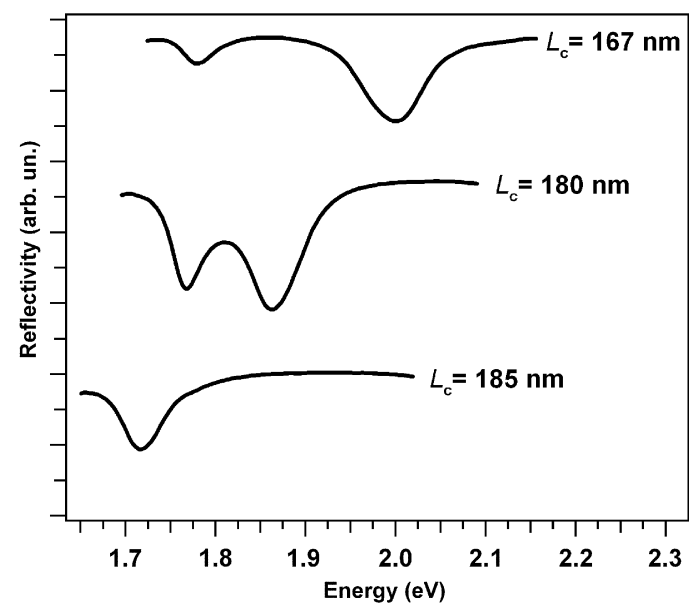

Fig. 2. Dependence of the reflection spectra of the organic-based monolithic microcavities on the thickness of the active layer (spectra acquired at normal incident angle). Spectra are vertically shifted for clarity.

ing the cavity length, $L_{\mathrm{c}}$, simply by varying the $J$-cyanine thickness in the range $167-185 \mathrm{~nm}$. Exploiting progressively thinner active layers, the blue-shift of the lower energy reflection dip slows down and the peak intensity decreases correspondingly. Vice versa, the blue-shift of the higher energy dip enhances, and its intensity increases upon decreasing the $J$-aggregate thickness. Overall, these results exhibit an anticrossing behaviour in accordance with the expected formation of cavity polaritons, indicating that the exciton-photon system is on resonance at normal incidence for $180 \mathrm{~nm}$-thick $J$-aggregate films. The resulting vacuum Rabi splitting is as large as $100 \mathrm{meV}$.

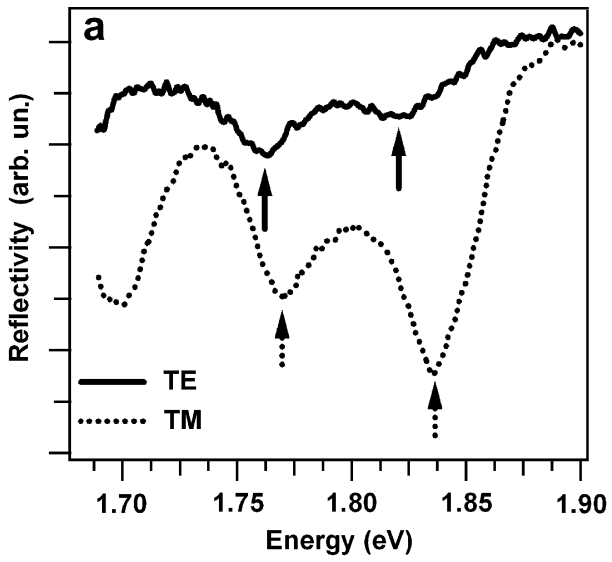

In addition, microcavity devices can exhibit polarization effects, such as, for example, polarization mode splitting, observed in the weak coupling regime in a number of organic-based microcavities $[21,22]$. In the present experiment, we have investigated the polarization state of the cavity polariton through both reflectivity and photoluminescence measurements. Polarization mode splitting arises from different penetration length, $L_{\mathrm{DBR}}$, into the dielectric mirror for $\mathrm{TM}$ and TE polarized e.m. fields. It depends on both the incident angle and the polarisation state of the incident light. Moreover, the resonance frequency $E_{\mathrm{m}}$ of a microcavity is dependent on the effective cavity length $L_{\text {eff }}$ $\left(L_{\mathrm{eff}}=L_{\mathrm{c}}+L_{\mathrm{DBR}}\right)$ :

$E_{\mathrm{m}}=\frac{L_{\mathrm{c}} E_{\mathrm{c}}+L_{\mathrm{DBR}} E_{\mathrm{s}}}{L_{\mathrm{eff}}}$

where $E_{\mathrm{s}}$ is the centre of the stop band. In the case of $E_{\mathrm{c}}=E_{\mathrm{s}}$, the microcavity resonance frequency is given by the Fabry-Perot energy $E_{\mathrm{c}}$, whereas, if $E_{\mathrm{c}}$ is different from $E_{\mathrm{s}}$, the resonance energy will be polarization dependent with an angular dependence of the resulting energy splitting given by [17]:

$E_{\mathrm{m}}^{\mathrm{TM}}(\theta)-E_{\mathrm{m}}^{\mathrm{TE}}(\theta)=A\left[E_{\mathrm{s}}(0)-E_{\mathrm{c}}(0)\right] \sin ^{2}(\theta)$

where $A$ is a constant.

Fig. 3(a) displays the typical reflectivity spectra acquired at a collection angle of $32^{\circ}$ with incident TM and TE polarized radiation. The LP (UP) peak is localised at $1.771(1.837)$ and $1.761(1.821) \mathrm{eV}$ for the TM and the TE polarization, respectively. A similar finding characterizes also the emission spectra (Fig. 3(b)), acquired at the same collection angle

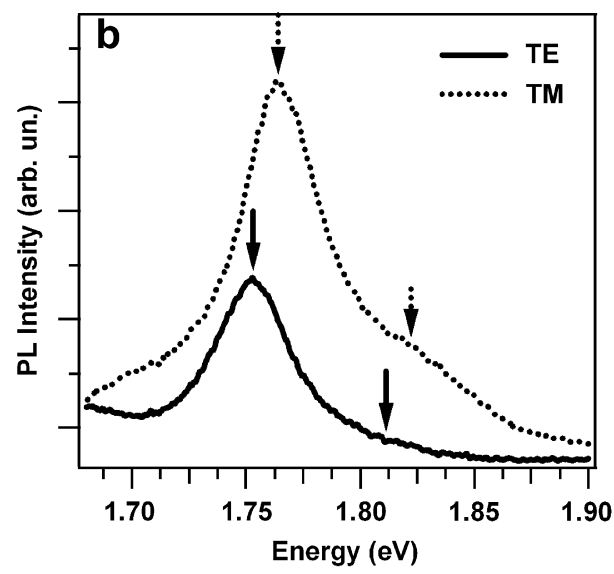

Fig. 3. TM (dotted line) and TE (continuous line) reflection (a) and photoluminescence (b) spectra. The position of the polariton peaks is evidenced by arrows. The acquisition angle was $32^{\circ}$. The peak at low energy is due to a DBR feature. 


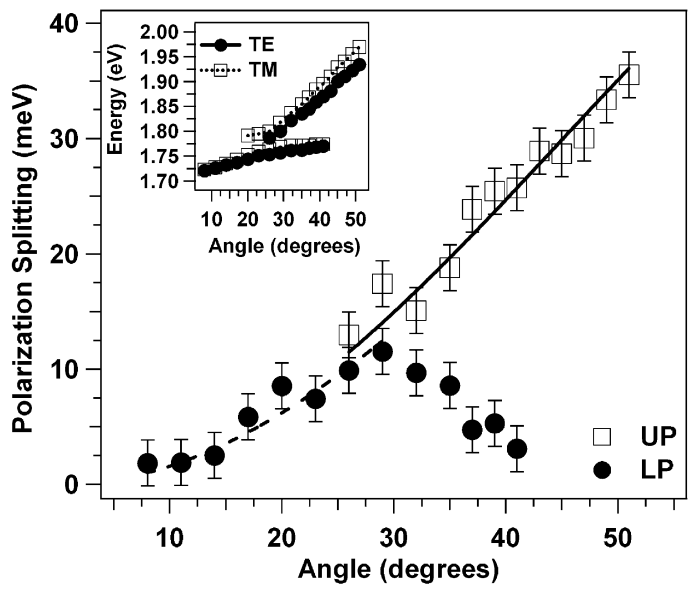

Fig. 4. Polarization splitting by reflectivity measurements, as a function of the incident angle for the LP (filled circles) and UP (empty squares) polariton branches. The dashed and continuous lines are fits to the data with a $\sin ^{2}(\theta)$ function. The inset shows the experimental TM and TE dispersion curves as a function of the incident angle.

$\left(32^{\circ}\right)$. Furthermore, in Fig. 3 the TM polarized peaks are shifted towards higher energies with respect to TE modes, because for the investigated devices the cavity mode energy is lower than the centre of the stop band (Fig. 1(b)). This effect is observed at any incident angle, as evidenced in the inset of Fig. 4, where we plotted the angular polariton dispersion for both TM and TE polarization, obtained by reflectivity measurements. This trend is also observed in the PL measurements, although a quantitative comparison is not straightforward because reflectivity and PL measurements are performed in different points of the same sample and local inhomogeneities of the PVA/ $J$-aggregate film $[12,18]$ can affect the emission spectra.

Finally, we note that the $\sin ^{2}(\theta)$ angular dependence of the polarization splitting combined with Eq. (2) provides a similar angular behaviour for the LP polariton at small angles and for the UP polariton at large angles [17]. This is confirmed by the analysis of the angular dependence of the polarization splitting of our microcavities (Fig. 4), showing a polarization splitting increasing as $\sin ^{2}(\theta)$ for the UP branch $\left(\theta \leqslant 35^{\circ}\right)$ and for the LP branch at lower angles $(0 \leqslant \theta \leqslant 20)$. The found value for the $A$ constant of Eq. (3) is $1.3 \pm 0.2$. In the angular range where mixed exciton-cavity modes are formed $\left(25^{\circ} \leqslant \theta \leqslant 40^{\circ}\right)$ a non-monotonic behaviour is observed for the LP energy upon increasing $\theta$. The measured trend is similar to the one found for
GaAs-based cavities with $\operatorname{In}_{0.13} \mathrm{Ga}_{0.87}$ As quantum well as active layer [17,23], a behaviour that has been theoretically modelled by a TMR model, taking into account the energy dispersion of the effective refractive index of the structure. We believe that a better understanding of the observed angular dependence can be accomplished by modelling our samples with a TMR model that also includes the energy dispersion of the effective refractive index for TE and TM polarized light [24] and birefringence effects reported in organic microcavities working in the strong coupling regime [18].

\section{Conclusions}

In conclusion, we fabricated and characterised microcavities with all-dielectric mirrors [14,15], based on $J$-aggregate active layers, which show strong coupling between the photon modes and the excitons of the organics. The characteristic anticrossing angular dispersion of the cavity polaritons was observed by both reflectivity and photoluminescence measurements, with a Rabi splitting in the range $30-100 \mathrm{meV}$. In addition, we measured a polarization splitting of the cavity polaritons as large as $35 \mathrm{meV}$, whose angular dependence is well described by the polarization mode splitting modelling for photon-like polaritons. A more quantitative model, describing also the angular range where mixed exciton-photon modes are formed, should also include the dispersion of the structure effective refractive index and birefringence effects of the active layer, which are known to significantly modify the emission properties of organic microcavities in the strong coupling regime $[18,25]$. Our results show that the strong coupling regime can be also achieved in symmetric microcavities with all-dielectric mirrors, and open the possibility of realizing novel optoelectronic devices based on the unique optical properties of polarized organic cavity polaritons.

\section{References}

[1] N. Tessler, G.J. Denton, R.H. Friend, Nature 382 (1996) 695.

[2] P.K.H. Ho, D.S. Thomas, R.H. Friend, N. Tessler, Science 285 (1999) 233.

[3] J.M. Lupton, R. Koeppe, J.G. Müller, J. Feldmann, U. Scherf, U. Lemmer, Adv. Mater. 15 (2003) 1471.

[4] E. Yablonovitch, Phys. Rev. Lett. 58 (1987) 2059.

[5] J.D. Joannopoulos, R.D. Meade, J.N. Winn, in: Photonic Crystals: Molding the Flow of Light, Princeton University Press, Cambridge, MA, 1995. 
[6] D.G. Lidzey, D.D.C. Bradley, M.S. Skolnick, T. Virgili, S. Walker, D.M. Whittaker, Nature 395 (1998) 53.

[7] D.G. Lidzey, D.D.C. Bradley, T. Virgili, A. Armitage, M.S. Skolnick, S. Walker, Phys. Rev. Lett. 82 (1999) 3316.

[8] N. Takada, T. Kamata, D.D.C. Bradley, Appl. Phys. Lett. 82 (2003) 1812.

[9] R.J. Holmes, S.R. Forrest, Phys. Rev. Lett. 93 (2004) 186404.

[10] M.S. Skolnick, T.A. Fisher, D.M. Whittaker, Semicond. Sci. Technol. 13 (1998) 645.

[11] R. Houdré, C. Weisbuch, R.P. Stanley, U. Oesterle, P. Pellandini, M. Ilegems, Phys. Rev. Lett. 73 (1994) 2043.

[12] D.G. Lidzey, A.M. Fox, M.D. Rahn, M.S. Skolnick, V.M. Agranovich, S. Walker, Phys. Rev. B 65 (2002) 195312.

[13] J.R. Tischler, M.S. Bradley, V. Bulovic, J.H. Song, A. Nurmikko, Phys. Rev. Lett. 95 (2005) 036401.

[14] J.-H. Song, Y. He, A.V. Nurmikko, J. Tischler, V. Bulovic, Phys. Rev. B 69 (2004) 235330.

[15] L.G. Connolly, D.G. Lidzey, R. Butté, A.M. Adawi, D.M. Whittaker, M.S. Skolnick, R. Airey, Appl. Phys. Lett. 83 (2003) 5377.

[16] L. Persano, P. Del Carro, E. Mele, R. Cingolani, D. Pisignano, M. Zavelani-Rossi, S. Longhi, G. Lanzani, Appl. Phys. Lett. 88 (2006) 121110.
[17] G. Panzarini, L.C. Andreani, A. Armitage, D. Baxter, M.S. Skolnick, V.N. Astratov, J.S. Roberts, A.V. Kavokin, M.R. Vladimrova, M.A. Kaliteevsky, Phys. Rev. B 59 (1999) 5082.

[18] D.N. Krizhanovskii, R. Butté, L.G. Connolly, A.I. Tartakovskii, D.G. Lidzey, M.S. Skolnick, J. Appl. Phys. 93 (2003) 5003.

[19] L. Persano, R. Cingolani, D. Pisignano, J. Vac. Sci. Technol. B 23 (2005) 1654.

[20] R. Houdré, J.L. Gibernon, P. Pellandini, R.P. Stanley, U. Oesterle, C. Weisbuch, J. O'Gorman, B. Roycroft, M. Ilegems, Phys. Rev. B 52 (1995) 7810.

[21] T. Virgili, D.G. Lidzey, D.D.C. Bradley, S. Walker, Synth. Met. 116 (2001) 497.

[22] L. Persano, E. Mele, R. Cingolani, D. Pisignano, Appl. Phys. Lett. 87 (2005) 031103.

[23] D. Baxter, M.S. Skolnick, A. Armitage, V.N. Astratov, D.M. Whittaker, T.A. Fisher, J.S. Roberts, D.J. Mowbray, M.A. Kaliteevski, Phys. Rev. B 56 (1997) R10032.

[24] R. Kumar, A.P. Singh, A. Kapoor, K.N. Tripathi, Opt. Eng. 43 (2004) 2134.

[25] R.F. Oulton, N. Takada, J. Koe, P.N. Stavrinou, D.D.C. Bradley, Semicond. Sci. Technol. 18 (2003) S419-S427. 